\title{
EL CONGRESO EXTRAORDINARIO DEL PSOE (septiembre de 1979)
}

Después de cuatro meses de dirección política provisional, la Comisión Gestora elegida en el XXVIII Congreso ordinario del PSOE - después de que la Comisión Política del Congreso aprobara una resolución en la que el partido quedaba definido ideológicamente como marxista, lo que provocó que el secretario general saliente, Felipe González Márquez, renunciara a presentarse a la reelección en el cargoconvocó el Congreso extraordinario del Partido Socialista Obrero Español para los días 28 y 29 de septiembre de 1979. La Comisión Gestora estaba compuesta por las siguientes personas: Ramón Rubial, Carmen García Bloise, Federico Carvajal, José Prat y Antonio García Duarte.

Dos objetivos principales tenía este Congreso extraordinario: el primero era la elaboración de una nueva resolución política; el segundo, la elección de una nueva Comisión Ejecutiva.

El clima de tensión y enfrentamiento político-ideológico entre los dos sectores nacidos antes del XXVIII Congreso ordinario (17, 18, 19 y 20 de mayo de 1979) se había recrudecido a lo largo de estos meses de transición entre Congresos. Recordemos brevemente las posiciones de ambos colectivos socialistas:

1. ${ }^{\circ}$ El sector denominado «moderado», encabezado por Felipe González y Alfonso Guerra, fundaba su disposición al abandono del término «marxista» en base a la necesidad de adecuar al máximo el funcionamiento político del partido en relación con su electorado (trabajadores y clases medias) para posibilitar una futura victoria en las urnas.

2. El sector denominado «crítico», compuesto por los elementos socialistas tradicionales del partido, contrarios a la supresión del marxismo del proyecto de definición ideológica del mismo. Insistía este sector en la necesidad de un reforzamiento del partido en lo referente al funcionamiento democrático y su organización federativa proporcionando más autonomía a las organizaciones federales. Luis Gómez Llorente, Francisco Bustelo y Pablo Castellano lideraban este sector.

\section{EL CONGRESO}

Asistieron a este Congreso extraordinario 421 delegados, distribuidos de la siguiente forma: 


\begin{tabular}{|c|c|c|c|}
\hline Andalucía $\ldots \quad \ldots$ & & 103 & delegados \\
\hline Aragón $\ldots \ldots \ldots$ & ... & 9 & $»$ \\
\hline Asturias $\ldots \ldots \ldots$ & ... & 23 & $\gg$ \\
\hline Baleares $\ldots \ldots \ldots$ & $\ldots$ & 4 & $\gg$ \\
\hline $\begin{array}{lllll}\text { Canarias } & \ldots & \ldots & \ldots\end{array}$ & $\ldots$ & 8 & $»$ \\
\hline Cantabria ... ... & $\ldots$ & 7 & $»$ \\
\hline $\begin{array}{lll}\text { Cataluña } & \ldots & \ldots\end{array}$ & $\ldots$ & 18 & 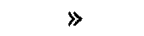 \\
\hline Castilla-La Mancha & $\ldots$ & 41 & $\gg$ \\
\hline Castilla-León $\quad \ldots$ & $\ldots$ & 22 & $\gg$ \\
\hline Euskadi $\ldots \ldots \ldots$ & $\ldots$ & 24 & $\gg$ \\
\hline Extremadura $\quad \ldots$ & $\ldots$ & 15 & $\gg$ \\
\hline $\begin{array}{llll}\text { Galicia } & \ldots & \ldots & \ldots\end{array}$ & $\ldots$ & 16 & $\gg$ \\
\hline Madrid $\ldots . .$. & $\ldots$ & 38 & $»$ \\
\hline
\end{tabular}

\begin{tabular}{|c|c|c|c|}
\hline Murcia & & 9 & delegados \\
\hline $\begin{array}{c}\text { País Valenciano } \\
\text { (25 comarcas })\end{array}$ & $\ldots$ & 63 & $\gg$ \\
\hline La Rioja ... ... & & 2 & $\gg$ \\
\hline Melilla ... ... ... & $\ldots$ & 1 & $\gg$ \\
\hline $\begin{array}{llll}\text { Ceuta } & \ldots & \ldots & \ldots\end{array}$ & $\ldots$ & 2 & $\gg$ \\
\hline Bélgica - Luxemburg & so. & 2 & $》$ \\
\hline Reino Unido ... & $\ldots$ & 2 & $\gg$ \\
\hline América $\ldots \ldots \ldots$. & $\ldots$ & 2 & $\gg$ \\
\hline Holanda - Dinamarc & & 2 & $\gg$ \\
\hline $\begin{array}{llllll}\text { Suiza } & \ldots & \ldots & \ldots & .\end{array}$ & $\ldots$ & 2 & $\gg$ \\
\hline Francia Norte ... & $\ldots$ & 2 & $\gg$ \\
\hline Francia Sur $\ldots \ldots \ldots$. & $\ldots$ & 2 & $\gg$ \\
\hline $\begin{array}{cccc}\text { Alemania } & \ldots & \ldots & \text {. }\end{array}$ & & 2 & $\gg$ \\
\hline
\end{tabular}

Además fueron invitados los representantes de los partidos socialdemócrata y socialista de los siguientes países: Alemania Federal, Austria, Bélgica, Chile, Dinamarca, Finlandia, Francia, Gran Bretaña, Italia, Israel, Portugal, Suecia, Venezuela (Acción Democrática). Junto a estas delegaciones asistieron también las siguientes: Sindicato de la Química de Suecia, Internacional de Mujeres Socialistas, Grupo Socialista del Parlamento Europeo, Unión de Partidos Socialistas de las Comunidades.

Se presentaron ponencias correspondientes a las siguientes delegaciones del partido: Córdoba, Sevilla, Zaragoza, Baleares, Cantabria, León, Palencia, Salamanca, Soria, Valladolid, Ciudad Real, Cuenca, Toledo, Barcelona, Gerona, La Marisma, Garraf, Tierra Alta-Ribera del Ebros, Euzkadi, Vizcaya, Badajoz, Galicia, Madrid, Murcia, Alicante, Baix Maestrat, Costeria, Horta Norte, Hoya de Buñol, Medio Vinalopo, Rincón de Ademuz, Valencia (estas ocho últimas todas del País Valenciano), Bélgica-Luxemburgo, Francia Sur y Africa, México y Reino Unido.

Después de las sesiones de discusión se aprobó la redacción final de la resolución política, cuyo punto más delicado - el referente al problema de la inclusión o no del marxismo en la definición ideológica - dice así: «El PSOE asume el marxismo como instrumento teórico, crítico y no dogmático para el análisis y transformación de la realidad social, recogiendo las distintas aportaciones, marxistas y no marxistas, que han contribuido a hacer del socialismo la gran alternativa emancipadora de nuestro tiempo y respetando plenamente las creencias personales.»

Pero si este reconocimiento del marxismo como instrumento de análisis y transformación pudiera interpretarse como una victoria del «sector crítico», los resultados de la votación para la elección de la nueva Comisión Ejecutiva no dejan lugar a dudas con respecto al peso real del «sector moderado» en la totalidad del partido socialista. E1 Congreso votó así: $85,9 \%$ de los votos para el «sector moderado»; $6,9 \%$ para el «sector crítico», y 7,2\% de abstenciones.

La nueva Comisión Ejecutiva del PSOE quedó configurada de la siguiente forma:

Presidente: Ramón Rubial (87,1\% de los votos).

Secretario general: Felipe González Márquez $(85,9 \%)$.

Vicesecretario general: Alfonso Guerra $(85,5 \%)$.

Secretatio de Organización: Carmen García Bloise $(85 \%)$.

Secretario de Administración: Emilio Alonso (83,3\%).
Secretario de Formación: José María Maravall $(81,2 \%)$.

Secretario de Estudios y Programas: Javier Solana Madariaga $(84,6 \%)$.

Secretario de Política Sectorial: Ciríaco de Vicente $(85,6 \%)$.

Secretario de Prensa y Propaganda: Guillermo Galeote (73\%).

Secretario de Política Municipal: Luis Fajardo $(73,4 \%)$. 
Secretario de Relaciones Políticas: Enrique Múgica Herzog (83,2\%).

Secretario de Relaciones con las Juventudes: Juan A. Barragán $(82,6 \%)$.

Secretario de Política Autonómica: María Izquierdo Rojo $(85,1 \%)$.

\section{VOCALES:}

José Federico de Carvajal (75,2\%).

José María Benegas $(86,1 \%)$.

Raimon Obiols $(86,2 \%)$.

Carlos Cigarrán $(84,7 \%)$.
Secretario de Política Sindical: Joaquín Almunia $(84,6 \%)$.

Secretario de Emigración: Francisco López del Real $(85,2 \%)$.

Secretario de Cultura: Ignacio Sotelo $(84,1 \%)$.
Gregorio Peces-Barba (73\%). Donato Fuejo Lago $(84,7 \%)$. Pedro Bofill $(84,7 \%)$.

Miguel Angel Villa $(84,5 \%)$.

Fernando Ollero Butler

Universidad Nacional de Educación a Distancia

\section{EXTRACTO DE LA RESOLUCION POLITICA APROBADA EN EL CONGRESO EXTRAORDINARIO DEL PSOE}

\section{II. estrategia y OBjetivos Politicos ACtuales}

\section{Nuestra estrategia democrática bacia el socialismo}

\subsection{La vía democrática al socialismo}

En la vía hacia la construcción de una sociedad socialista es preciso conquistar el poder político por la clase trabajadora y los diversos sectores sociales que se aglutinan en torno a ella, y que estando y sintiéndose interesados en el cambio de sociedad pueden desplazar a las clases dominantes que se han garantizado a través de ese poder político su propio predominio social.

Es necesario, por tanto, conquistar el poder político para alterar la relación social de fuerzas y conquistarlo democráticamente en coherencia con los principios que inspiran la acción política del socialismo. Hemos de sumar al ideal socialista y a la lucha por este ideal a la más amplia mayoría de la población, pues el triunfo del socialismo dependerá de que los socialistas consigan, en el proceso dialéctico de formación de las condiciones objetivas para el cambio, que la mayoría de los ciudadanos se convierta en una mayoría política consciente, que luche decididamente, utilizando siempre los medios democráticos, por lograr la transformación de la sociedad.

Esta conquista del poder político ha de derivar, pues, de que el PSOE obtenga el apoyo popular suficiente como para poder acceder a partir de la formación de una mayoría parlamentaria al gobierno del Estado.

La consecución de esta mayoría parlamentaria basada en el sufragio universal es condición necesaria para iniciar el período de construcción de la sociedad socialista y lo es, ante todo, porque está íntimamente ligada a la cultura política y social de las sociedades occidentales. Al objeto de conseguir una mayoría parlamentaria el PSOE procurará captar la mayoría del electorado cuyos intereses coinciden objetivamente con nuestros planteamientos, mediante la difusión del mensaje que nos es 
propio, de acuerdo con los programas aportados por el Partido y utilizando las campañas electorales como etapas en las que se intensifica la conciencia de los trabajadores y de los demás sectores oprimidos.

\subsection{Profundización y ampliación de la democracia}

Para el PSOE no sólo el socialismo es sinónimo de democracia y libertad, sino que además la estrategia para alcanzar el socialismo consiste en la profundización y la ampliación de la democracia. Nuestra vía es la vía democrática al socialismo basada en la voluntad y la lucha de la gran mayoría.

$\mathrm{El}$ avance hacia el socialismo exige unas condiciones políticas que lo hagan posible: condiciones institucionales y condiciones sociales. La «revolución de la mayoría» de la que ya hablaba Engels implica, pues, una política de masas, una política en la que los socialistas, como fuerza mayoritaria de los trabajadores y del pueblo, hemos de impulsar la creación de un amplio y mayoritario bloque social contra la dominación capitalista, rechazando el vanguardismo y el sectarismo e impulsando la unidad social de una amplia mayoría. La revolución no es el «gran día», sino el proceso permanente y conflictivo para generar el cambio de sociedad. Este proceso de cambios, sean radicales o graduales, de la sociedad y el Estado pone de forma permanente sobre la mesa el tema del poder burgués. $Y$, por tanto, la cuestión de valorar nuestros objetivos tácticos en función de la relación de fuerzas. Para alcanzar el poder es preciso contar con el apoyo y la participación de una nueva mayoría en una revolución lenta de la sociedad dirigida en tres direcciones: en el terreno político-institucional (reforzando, ampliando y profundizando la democracia y las libertades), en el terreno económico (ampliando el área de participación y decisión colectiva en los centros de poder económico) y en el campo de la sociedad civil (creando nuevas formas de organización cultural de la sociedad, impulsando la hegemonía social de los trabajadores y del pueblo). Los progresos no serán lineales, ni de golpe, ni simultáneos, ni los podemos dejar a la espontaneidad de cada sector. El socialismo es también precisamente esto: este proceso histórico de enfrentamiento contra la dictadura social de los capitalistas y la aparición germinal de elementos de la nueva sociedad. Corresponde a los trabajadores, y por tanto a los socialistas, situarlo bajo su dirección de clase y darle unos objetivos a corto, medio y largo plazo para evitar los peligros de una «situación catastrófica» o de una «falsa posición» de los socialistas $\mathrm{e}$ ir transformando la relación de fuerzas sociales existentes.

Una condición indispensable para el avance hacia el socialismo será la articulación, en torno a un objetivo común, de todos los sectores sociales interesados en la construcción de la nueva sociedad. Nuestra estrategia deberá centrarse en la formación de un amplio bloque de clases, agente de las transformaciones revolucionarias, y en la afirmación de su protagonismo constante en la lucha por la democracia y el socialismo.

Esta tarea deberá partir del reconocimiento de la diversidad esencial existente en el seno de la clase trabajadora y, al mismo tiempo, de la identidad fundamental de intereses de las diferentes fracciones y capas que la integran. Porque mientras los trabajadores industriales continúan su proceso de crecimiento y ampliación, aparecen nuevos sectores que venden su fuerza de trabajo y se insertan en el proceso productivo en una posición subordinada (técnica, administrativos, trabajadores no directamente vinculados a la producción). En un ritmo creciente también los intelectuales ven frustrada su vocación creadora en el seno del capitalismo; cada día son más numerosos los profesionales asalariados, y un número cada vez mayor de pequeñas empresas son conducidas a la crisis por el capital monopolista, lanzando al mercado de trabajo a los grupos sociales que las impulsaban; los campesinos, por su parte, se encuentran ante el dilema de agotarse en unas condiciones de empobre- 
cimiento creciente, o bien de constituirse en mano de obra barata para la industria del interior o del extranjero. En el seno de todos estos grupos de trabajadores se produce una lucha entre la influencia burguesa (fundamentalmente ideológica a través de la alienación cultural y consumista) y las fuerzas del socialismo que van avanzando en la práctica social, ideológica y política, modificando la relación de fuerzas en su favor, pues estos grupos sociales se ven progresivamente enfrentados con los intereses capitalistas.

A pesar de las contradicciones y divisiones que se producen en su seno, siempre instrumentalizadas por la burguesía y condicionadas por diversos factores (lugar en el proceso productivo, niveles de renta, etc.); todo este diversificado conjunto de fracciones y capas sociales forma ya un bloque de intereses que hay que cohesionat progresivamente porque sus aspiraciones tienden a coincidir en un proyecto de creación de una sociedad en la que se haya eliminado la opresión y la explotación de clase. Es decir, una sociedad socialista.

Al PSOE incumbe, asumiendo su responsabilidad como alternativa mayoritaria, la. impulsión y formación de este nuevo bloque social, centrado por los sectores más concienciados en la lucha contra el capitalismo (los trabajadores industriales) y basado en la articulación de un amplio movimiento de masas.

Dada la existencia de contradicciones entre los distintos sectores sociales que se pueden integrar en el bloque de clases, la unificación de todos ellos tiene un carácter de compromiso: el compromiso por el socialismo. La profundización en el estudio de características y situaciones, de las aspiraciones e intereses de los distintos sectores que componen el bloque de clases será la vía para hacer posible un compromiso sobre alternativas programáticas que han de ir concretizándose y pormenorizándose paulatinamente. A través de la adopción unitaria de un proyecto político socialista, el bloque de clase se convertirá en el protagonista del proceso de construcción del socialismo.

El compromiso por el socialismo se concreta también en un compromiso geográfico. Las diferencias y desequilibrios interterritoriales exigen de los socialistas una respuesta programática que esté dirigida, contemporáneamente, a la superación global de la crisis y a la superación de las diferencias existentes entre las nacionalidades y regiones de España. La diferente situación de desarrollo en que se encuentran obliga, en efecto, a hacer una política que esté dirigida primordialmente a satisfacer las exigencias de las nacionalidades y regiones menos favorecidas, al mismo tiempo que se satisfagan las necesidades de los sectores sociales oprimidos en todo el ámbito del Estado.

\subsection{Contenido social $e$ institucional de la estrategia socialista}

La estrategia socialista combina un contenido institucional y un contenido social; una lucha en el interior de las instituciones del Estado para hacer más real y viva la democracia y una lucha social por impulsar la participación del pueblo y su hegemonía en la sociedad civil. Los socialistas hemos de conseguir la hegemonía social de los trabajadores, impulsando la vertebración de la sociedad civil. Por eso es necesatio que los socialistas nos insertemos más y más en el tejido social. La posición de los socialistas, como partido de los trabajadores más solidariamente implantado en el terreno electoral, nos obliga ahora a avanzar más en el camino de la vertebración social del pueblo, siendo una fuerza política presente en todas partes y expresiva de las reivindicaciones populares en el marco de un programa concreto de transformaciones para que el apoyo electoral pueda ser materializado en una política de gobierno socialista. Los socialistas hemos de sintentizar las diversas aspiraciones de cambio de cada uno de los sectores sociales dominados tanto en el programà como en la actuación conectándolos con lạ lucha política dentro y fuera 
del Estado, poniendo siempre de relieve los intereses generales y políticos de los trabajadores e impulsando la unificación de las reivindicaciones anticapitalistas bajo un planteamiento general.

Esta estrategia combinada de lucha institucional y social es la que puede permitir en España el desarrollo de una línea global que haga posible el acceso de los socialistas a la dirección política del pueblo y a los trabajadores a la hegemonía democráticamente establecida sobre la nueva mayoría social. Esto sólo es posible transformando profundamente el Estado heredado del pasado, creando centros de poderes diversificados que debiliten la concentración de poder en que se ha basado el dominio de la burguesía española, impulsando la democracia en todas partes como factor de debilitación del poder de la minoría dominante. Por eso los socialistas nos definimos como el partido de las autonomías, como los impulsores más decididos y consecuentes de un Estado basado en las comunidades autónomas. Esto será un paso de importancia histórica trascendental en el camino de la construcción de una estructura federal del Estado basado en el autogobierno y la libertad de sus pueblos. Pero es también un elemento clave en la construcción y consolidación dinámica del Estado democrático, que permite una dialéctica combinada de poder central y comunidades autónomas, el acceso desigual y arrítmico a centros de poder para potenciar, por un lado, la capacidad hegemónica de los socialistas y, por otro, el impulso de los sectores donde las posiciones se hallan más retrasadas. Solamente estructurando la democracia sobre la base de la construcción de una estructura de autogobiernos de las nacionalidades y regiones de España podemos avanzar en el debilitamiento de los instrumentos de dominación tradicionales de los terratenientes y la burguesía española e impulsar la alternativa emancipadora de los trabajadores.

Los avances en esta lucha por la creación de un Estado democrático bajo el impulso de una nueva mayoría social del progreso y su dirección por los trabajadores hará posible crear las bases para pasar al socialismo. La nueva fase que deja abierta la lucha de clases en España hace que los socialistas como partido de los trabajadores seamos hoy también el partido de la democracia, el partido de las autonomías, el partido que expresa e impulsa la voluntad del pueblo para cambiar de sociedad.

\section{Objetivos actuales del PSOE}

\subsection{Defensa de los intereses de los trabajadores y apoyo a la UGT}

Como partido de los trabajadores, los socialistas nos planteamos en primer lugar la defensa de sus intereses concretos e inmediatos. $Y$ apoyamos en este sentido, de forma total, la acción de la Unión General de Trabajadores, el sindicato socialista. Al mismo tiempo, los socialistas tenemos igualmente por objetivo la defensa de los intereses globales, políticos, de los trabajadores. Este doble objetivo de los socialistas, dentro del partido y dentro del sindicato, refuerza y estrecha más los vínculos fraternales entre el partido y la UGT y comporta la combinación permanente entre el combate sindical y el político. Esto implica hoy en especial, como tarea del partido y de los socialistas, la consolidación y el reforzamiento de la UGT y el desarrollo de una política global destinada a combatir el paro, la depreciación de los salarios, el deterioro de las condiciones de vida del pueblo. Es decir, el desarrollo de una política que permita defender los intereses populares en una etapa de crisis económica capitalista de un alcance hasta hoy desconocido.

Para los socialistas, una de las condiciones básicas para el desarrollo y la consolidación de la democracia es la necesaria existencia de unos sindicatos fuertes y 
consolidados. En estos momentos este objetivo requiere desarrollar en profundidad los derechos sindicales amparados por la Constitución - negociación colectiva, acción sindical en la empresa, Estatuto del Trabajador, huelga, etc.-, así como la devolución a las centrales sindicales de clase el patrimonio sindical, y en concreto a la UGT el patrimonio histórico que le fue arrebatado en el año 1939. Esta acción debe encaminarse a lograr que la democracia penetre en las empresas, en la perspectiva de un avance de los trabajadores hacia mayores parcelas de poder.

La acción de los socialistas en el seno del movimiento sindical -en concreto en la UGT - debe ir encaminada a dotar de contenido político a las luchas reivindicativas y a conseguir elevar la conciencia política de los trabajadores. Los militantes socialistas contribuirán a desarrollar un amplio debate en sus áreas de acción sindical, aportando elementos de análisis y fomentando el debate autónomo de los sindicatos, a través de la potenciación de sus mecanismos democráticos internos y del respeto escrupuloso de los acuerdos adoptados. Esto significa en la práctica el rechazo a todo control burocrático, organizativo o administrativo desde el partido, es decir, en definitiva, el rechazo del sindicalismo concebido como correa de transmisión del partido. Del mismo modo, y a la inversa, los socialistas rechazamos también las prácticas «tradeunionistas», concebidas como control del sindicato sobre el partido. Mantenemos, sin embargo, la conveniencia de conjugar esfuerzos para la consecución de nuestro común objetivo de construcción de la sociedad socialista.

En coherencia con su política de defensa de los intereses de todos los trabajadores, el PSOE exige la elaboración de un Estatuto de la Función Pública que garantice la defensa de los intereses de todos los funcionarios y su independencia de la Administración.

\subsection{Desarrollo progresista de la Constitución}

La Constitución de 1978 es la expresión formal del final del sistema legal franquista, la consagración de un régimen de libertades, el afianzamiento de un sistema democrático basado en el sufragio universal. Los socialistas, en tanto que fuerza más consecuentemente democrática, somos hoy en España el partido de la democracia. Los preceptos constitucionales que garantizan la libertad y la igualdad políticas han abierto nuevas posibilidades a los trabajadores para organizarse, para luchar en mejores condiciones por la dirección de la sociedad, para oponerse a la ideología burguesa, para aprovechar las contradicciones internas de la clase burguesa. En esta perspectiva, el avance de la democracia es un objetivo actual y fundamental del socialismo, como lo es la lucha contra todas las tentativas de desestabilizarla o de reducir su alcance. Esto significa, en primer lugar, velar por el respeto íntegro de la Constitución y avanzar en la democratización del Estado y de la sociedad, haciendo realidad todo el contenido democrático del texto constitucional. No será cosa fácil ni cosa de un día: significará enfrentarse a la tarea de la transformación democrática del Estado. Las Leyes Orgánicas que habrán de elaborarse en un inmediato futuro son ya el primer paso en este camino de confrontación, en la que se enfrentarán una concepción conservadora y estática y una concepción progresista avanzada.

\subsection{Democratización del Estado}

Por otra parte, los socialistas tenemos plena conciencia de que los aparatos del Estado han servido hasta hoy a los intereses de la clase dominante y que en España siguen siendo escasamente democráticos, como consecuencia de las peculiaridades 
del proceso del tránsito de la dictadura franquista a la situación actual. Por ello la lucha por la democratización de los aparatos del Estado es hoy una perspectiva táctica crucial. Desde su acción opositora, enérgica y responsable, el Partido Socialista debe luchar constantemente para hacer transparentes las actividades de los aparatos estatales y de los distintos organismos públicos, impulsando todas las iniciativas que permitan ir acabando con los residuos burocráticos del franquismo, suprimir corrupciones y privilegios, democratizar paulatinamente los entresijos de la Administración y exigir en todo momento el más estricto respeto a la Constitución y a los principios democráticos.

La huella que ha dejado en nuestra sociedad el paso de sucesivos regímenes autoritarios, el último de los cuales fue el franquismo, explica que debamos dedicar una atención especial a la liquidación de la herencia autoritaria en el seno de nuestra sociedad. Los socialistas debemos utilizar todos los medios para conseguir aquella imagen de honradez y capacidad que pueda conseguir la adhesión de la mayoría, fomentando las actitudes solidarias y haciendo que todos puedan sentirse implicados en la tarea colectiva. Los socialistas debemos impulsar una acción de revitalización política de la población y una amplia campaña de fomento de cualquier tipo de actividad cultural, deportiva, etc., en el camino hacia la creación de otros tipos de valores que tiendan al asentamiento democrático y el avance hacia el socialismo.

El combate por las autonomías de las distintas nacionalidades y regiones es también uno de nuestros esenciales objetivos prioritarios. Los socialistas, que impulsamos el creciente protagonismo hegemónico de los trabajadores en los procesos de afirmación nacional y regional en el Estado, lucharemos por la consecución de unos Estatutos de Autonomía de la máxima amplitud, sin discriminaciones de ningún género.

\subsection{El PSOE, primer partido de la oposición}

A un nivel político general, los socialistas constituimos hoy en España la principal fuerza política de los trabajadores y el principal partido de la oposición. Esta situación exige al partido una doble dimensión en su actuación táctica. Debe ejercer, por un lado, una tarea enérgica de oposición con el objetivo de conseguir un desarrollo auténticamente democrático del marco constitucional, denunciar e impedir cualquier intento de involución autoritaria del proceso democrático, controlar al poder ejecutivo y defender en todo momento los intereses y las aspiraciones de los trabajadores. Pero, por otro lado, las especiales responsabilidades del partido, como alternativa efectiva y posible de poder, obligan a un esfuerzo permanente para afinar sus propuestas programáticas, potenciar sus energías dirigentes y ampliar progresivamente, en los planos políticos organizativos y técnicos, su capacidad efectiva de dirección de la sociedad y del Estado. Las situaciones derivadas del incremento de la violencia y del terrorismo y de eventuales intentos de desestabilización del proceso político, así como las graves dificultades suscitadas por la ampliación de la crisis económica, obligan más aún, si cabe, a ampliar esta dimensión de las responsabilidades de oposición y de partido con vocación de gobierno. El partido debe ser consciente de que existen unos límites para su actuación, impuestos por la correlación de fuerzas y las dificultades de tipo político y económico a las que se enfrenta el proceso democrático español, pero debe ser consciente también de que esas situaciones pueden ser a su vez aprovechadas por la clase dominante y sus expresiones políticas para mellar nuestra fuerza opositora, para frenar nuestro avance. En particular, el Partido Socialista debe velar ante todo para evitar cualquier intento de involucrarlo en una corresponsabilización subordinada en la gestión de los 
problemas actuales. Tan sólo ante eventos de extrema gravedad para la perduración del sistema democrático, el Partido Socialista debería consentir el sacrificio de aportar su colaboración a una política de estricta defensa del marco democrático en colaboración con las demás fuerzas democráticas. Pero el riesgo que para el partido comportaría esta eventualidad es tan considerable que obliga a rechazarla salvo en casos de estricta supervivencia del proceso constitucional y democrático, en cuyo caso debería procederse a una muy severa acotación de los límites temporales y de los contenidos programáticos.

En circunstancias de normalidad democrática, el PSOE ha de mantener una política autónoma e independiente, reflejo del carácter diferenciado de la alternativa socialista que representa. Cualquier colaboración temporal con otras fuerzas políticas deberá ser sometida y aprobada por el Comité Federal del partido, sin que en ningún caso los acuerdos en que se concrete el compromiso signifiquen un retroceso en el camino hacia los objetivos del programa máximo.

\subsection{La lucba contra el terrorismo y la violencia}

Uno de los principales objetivos del PSOE debe ser el establecimiento de una correcta política de orden público que contribuya a hacer desaparecer el creciente sentimiento de inseguridad ciudadana, que constituye una de las amenazas más grandes que se cierne sobre nuestra naciente democracia. El PSOE condena tajantemente el terrorismo cualquiera que sea su signo y no admite exista dentro de un contexto democrático causa alguna que justifique tal medida de actuación que sólo contribuye a la desestabilización del país. Considera que en la defensa del Estado en su lucha contra el terrorismo no sólo hay que adoptar medidas policiales y coercitivas, sino también medidas políticas que supongan implicar a todos los sectores de la sociedad española en el objetivo común de erradicar la violencia política, único medio de aislar a los terroristas de la sociedad y de aumentar y fortalecer las convicciones de todos los ciudadanos. La violencia y el aumento de la delincuencia común que se observa en nuestros pueblos y ciudades, que están siendo en muchos casos instrumentalizadas por las fuerzas más reaccionarias del país, requiere un análisis pormenorizado de sus causas -entre las cuales el aumento de paro y de la frustración juvenil por no encontrar empleo es una de las principales- para atacar en su raíz este problema, así como el establecimiento de una política penitenciaria y asistencial que permita reducir los niveles de violencia que engendran inseguridad. Dicha política penitenciaria y asistencial deberá ir dirigida a la rehabilitación y reinserción del recluso en la sociedad.

\subsection{Las autonomías}

EI PSOE defenderá e impulsará el desarrollo constitucional de las autonomías con objeto de alcanzar una nueva configuración del Estado, que posibilite el autogobierno de los pueblos, basado en la exigencia de la solidaridad entre las distintas nacionalidades y regiones, que evite inadmisibles desequilibrios territoriales entre ellos.

El PSOE considera que el proceso autonómico de las nacionalidades y regiones no es la consecuencia coyuntural de un pacto democrático establecido con anterioridad a la desaparición de la dictadura, sino que obedece a un sentimiento real de los pueblos, nacionalidades y regiones que configuran el Estado.

El partido entiende que sólo un análisis amplio de las profundas razones históricas, culturales y económicas y sociales de todas y cada una de las nacionalida- 
des y regiones de España propiciará la adhesión de la población a la existencia de comunidades autónomas, aceptando el desarrollo de proyectos de sociedad en consonancia con sus peculiaridades y dentro del marco general del Estado. El partido propiciará que la solidaridad entre los pueblos de España sea el más vivo reflejo de la solidaridad de la clase trabajadora.

Los órganos de dirección del partido promoverán el desarrollo autónomo de las organizaciones socialistas del Estado, asumiendo y sintetizando los objetivos comunes.

La descentralización autonómica que se propone para el Estado encontrará su reflejo en el seno del partido procurando una distribución equilibrada de las competencias políticas, financieras y administrativas que permita el real desarrollo y avance de los partidos nacionales y federaciones que integran el Partido Socialista Obrero Español.

Así, pues, el PSOE reafirma en este punto su vocación autonómica en el convencimiento de que sólo la consolidación de la democracia y el afianzamiento de una estructura administrativa del Estado descentralizada, en el marco de lo que son los entes autonómicos, sentarán las condiciones objetivas para la transformación de un Estado centralista en el Estado Federal que el PSOE propugna.

\subsection{La acción municipal}

Frente a unas colectividades locales en permanente crisis, enfrentadas a una concentración anárquica de medios y de personas en las grandes ciudades y al minifundismo y depauperación acelerada en las zonas rurales (lógica consecuencia de una sociedad insolidaria y de un sistema político centralista y autoritario), los socialistas debemos implantar una política municipal nueva que suponga una reforma en profundidad de las estructuras, competencias y funcionamiento de los Ayuntamientos y Diputaciones y constituya el primer salto cualitativo hacia el proyecto de cambio de la sociedad que proponemos.

La democratización plena de nuestra vida pública depende notoriamente de una reforma profunda de la Administración Municipal, que debe suponer un cambio real en la vida de los pueblos y ciudades de nuestro país. A tal fin, el PSOE luchará por aumentar los recursos de las Haciendas locales, reformando los sistemas de fiscalidad, aumentando la financiación y subvención del Estado y organismos crediticios, introduciendo a la vez el principio de transparencia y control democrático de su gestión. Igualmente, el PSOE exige la promulgación de una nueva Ley de Régimen Local y disposiciones complementarias, así como la reelaboración de una nueva política del suelo urbano y de ordenación del territorio.

Los Ayuntamientos se abrirán al pueblo que, a través de sus iniciativas, es quien debe definir la vida municipal. La participación en Asociaciones de Vecinos, el apoyo a las mismas y la descentralización y delegación de facultades en los barrios son objetivos de la estrategia municipal socialista. Junto a ello, el PSOE realizará un esfuerzo para que se aumenten las competencias de los municipios y su autonomía jurídico-económica.

En el ámbito rural, el PSOE fomentará la asociación de pequeños municipios para convertirlo en sujetos capaces de asumir las mismas responsabilidades y competencias que propugnamos para núcleos urbanos más importantes. Desde la Administración local, el PSOE apoyará las acciones mancomunadas que signifiquen una más fácil racionalización de los servicios y el ejercicio de criterios solidarios.

La actividad municipal de los concejales y alcaldes socialistas deberá coordinar la necesaria autonomía funcional con las directrices políticas emanadas de los órganos del partido, que apoyarán cotidianamente la labor de aquéllos a través de los 
servicios técnicos y asesorías que el partido promoverá en los ámbitos locales de su organización, así como mediante la movilización ciudadana y la participación de los militantes cuando fuera necesario. Así se evitará que la presencia socialista sea una mera gestión acertada de los intereses capitalistas en nuestros municipios.

$\mathrm{La}$ acción municipal de los socialistas deberá inscribirse, finalmente, en un marco de respeto a los compromisos municipales establecidos con todas las fuerzas progresistas. 